\title{
LA TRAYECTORIA POLÍTICA DE INDALECIO PRIETO DURANTE LA SEGUNDA REPÚBLICA: UN ESTADO DE LA CUESTIÓN
}

\author{
Javier RODRÍGUEZ IGLESIAS \\ Universidad de Salamanca
}

\begin{abstract}
RESUMEN: Indalecio Prieto ha sido uno de los políticos españoles contemporáneos que mayor interés ha despertado entre los historiadores, sobre todo en aquellos que se han acercado al estudio de la Segunda República. Este artículo aborda la evolución de la historiografía existente sobre este dirigente socialista a lo largo de las últimas décadas, estableciendo sus alcances y limitaciones. En primer lugar se acomete el estudio de Prieto dentro de la Historia del movimiento obrero y del socialismo español, para pasar a analizar aquellas obras que estudian su trayectoria política personal, centrándonos en aquellas que hacen referencia a la Segunda República, ámbito cronológico en el que hemos centrado nuestras investigaciones.
\end{abstract}

PALABRAS CLAVE: Indalecio Prieto, política, España, Segunda República, movimiento obrero, socialismo, PSOE, UGT.

ABSTRACT: Indalecio Prieto has been one of the contemporary Spanish politicians who has arisen great interest among historians, especially among those who have studied the Second Spanish Republic. This work aims to analyze the evolution of the existing Historiography about this socialist leader during the last decades, and to establish their scopes and limitations. First of all, the study of the figure of Indalecio Prieto within the Labour Movement and the Spanish Socialism is presented, and then we analyze the works that have studied his political career, specially those works that are focused on the Second Spanish Republic, historic period in which our research is centred.

KEYWORDS: Indalecio Prieto, Politics, Spain, The Second Spanish Republic, The Labour Movement, Socialism, The Spanish Socialist Party (PSOE), The General Union of Workers (UGT).

\section{INTRODUCCIÓN:}

Indalecio Prieto ha sido uno de los políticos españoles contemporáneos que mayor interés ha despertado entre los científicos sociales que se han acercado al estudio de la Historia de España del siglo XX, sobre todo de aquellos que lo han hecho a la Segunda República. Son numerosos y cualificados los historiadores, 
politólogos y sociólogos que han estudiado la trayectoria política de este dirigente socialista, ante todo su papel durante la etapa republicana.

Prieto ha sido analizado principalmente desde dos vertientes: la primera de ellas se corresponde con el análisis de su papel dentro de la dinámica de las organizaciones socialistas durante las décadas en las que fue un destacado dirigente de las mismas -principalmente durante los años veinte y treinta del siglo pasado-, y la segunda hace referencia a su destacada actuación política personal tanto durante la Dictadura de Primo de Rivera, como durante la Segunda República y la Guerra Civil. Conviene recordar que durante estos dos últimos períodos históricos desempeñó varios cargos ministeriales que le proporcionaron una gran notoriedad pública.

Las obras que han estudiado a Prieto dentro del contexto de la Historia del movimiento obrero español y, más en concreto, en el marco de la evolución del socialismo español durante las primeras décadas del siglo XX, fueron realizadas, en su inmensa mayoría, durante las décadas de los años setenta y ochenta del pasado siglo, principalmente durante la segunda mitad de los años setenta y la primera de los ochenta. Sin embargo, durante la década de los noventa y en los primeros años del nuevo siglo no han dejado de publicarse estudios sobre la evolución del socialismo español durante las primeras décadas del siglo XX y sobre la trayectoria política de Prieto.

Por otra parte, la mayoría de las obras monográficas que estudian su carrera política y su papel durante la Segunda República se publicaron en los primeros años de la década de los ochenta, principalmente en 1983, por motivos que analizaremos más adelante.

Las principales razones de esta cronología son varias y tienen mucho que ver con la situación política de la España de aquellos años ${ }^{1}$. Desde mediados de la década de los sesenta se vivió una etapa decisiva en la historiografía española.

1 PASAmar, G. (2000). La Historia contemporánea: Aspectos teóricos e historiográficos. Madrid: Editorial Síntesis, pp. 217-248. En estas páginas el autor analiza la evolución de la historiografía española sobre Historia Contemporánea durante el siglo XX. Para lo que en este trabajo nos concierne resulta interesante la parte en la que se centra en la historiografia española contemporánea desde la década de los sesenta en adelante.

Otras obras importantes para conocer las grandes líneas historiográficas del siglo XX, esta vez a nivel planetario, son: IGGERs, G. (1998). La ciencia histórica en el siglo XX. Las tendencias actuales. Una visión panorámica crítica del debate internacional. Barcelona: Idea Universitaria. y Moradiellos, E. (1992). Las caras de Clio. Introducción a la Historia y a la Historiografía. Oviedo: Universidad de Oviedo. 
Durante esta fase se llevaron a cabo los cambios políticos, sociales, intelectuales y profesionales que desembocaron en la historiografía actual. Aumentó el interés por la Historia Contemporánea, (hasta aquellos momentos muy escaso), se intentó combatir los tópicos historiográficos franquistas, potenciar la monografía local y tener una visión más amplia de la Historia, inspirada, sobre todo, en la Historia Económica y Social, siendo esta última la que tuvo la primacía en los estudios de Historia Contemporánea durante los años sesenta y sobre todo en los setenta. Las claves intelectuales que provocaron la consolidación de la Historia Contemporánea durante estos años residen en el interés por analizar las causas que provocaron el atraso español del siglo XIX y la tragedia española del siglo XX. Esto hizo que se despertara la curiosidad por los siglos XIX y XX. El interés por el siglo XX deriva, por lo tanto, de la meditación sobre el significado y las causas de la Guerra Civil, emprendida sobre todo por historiadores exiliados e hispanistas que han examinado la Historia Contemporánea española buscando las peculiaridades que llevaron al enfrentamiento civil. Esta reflexión intelectual se ha reflejado en la historiografía profesional a través de los hispanistas anglosajones y de Manuel Tuñón de Lara, que constituyen un punto de referencia esencial para la historiografía española de los años sesenta y setenta. El precursor de los hispanistas anglosajones fue Gerarld Brenan que consideraba que España era incapaz de articularse como nación moderna y que estaba lastrada por el clero, el ejército y los terratenientes, con una tendencia al localismo y a las insurrecciones. Abarcó la Historia política de la Restauración, de la Dictadura y de la República y, dentro del ámbito de la Historia Social, analizó el movimiento obrero socialista y anarquista. Los diversos hispanistas anglosajones de los años cincuenta y sesenta han ido formando grupos de investigación, siendo el más importante el de Raymond Carr.

Durante las décadas de los sesenta y los setenta principalmente, abundaron los estudios sobre la evolución del movimiento obrero en Europa debido a la importancia que le procuraron al estudio de los movimientos sociales y, dentro de este campo, al conocimiento del movimiento obrero las grandes corrientes historiográficas dominantes en aquellos años: principalmente la Escuela de los Annales y el marxismo.

El hecho de que la mayor parte de los estudios sobre el movimiento obrero español se realizaran con un cierto retraso cronológico respecto al resto de la Europa occidental se debe, principalmente, a la pervivencia del régimen franquista hasta mediados de los setenta. Hasta la década de los sesenta no se había realizado en España la Historia del movimiento obrero. En un principio esta nueva disciplina historiográfica representó una renovación dentro del ambiente historiográfico de la Dictadura de Franco, pero muchos de esos estudios, aunque tenían una notable calidad científica, estaban demasiado ideologizados e impregnados por un carácter 
militante contra la dictadura franquista. Realizar la Historia del movimiento obrero español, del movimiento socialista en particular y de sus principales líderes y portavoces suponía, en gran medida, oponerse al régimen de Franco. ${ }^{2}$

En los estudios que se han realizado sobre Prieto hay una fecha clave a destacar, 1983. En este año se conmemoró el centenario de su nacimiento y por este motivo se publicaron numerosas monografías sobre su carrera política. Fueron muy abundantes los libros $\mathrm{y}$, sobre todo, los artículos publicados en revistas especializadas y en diversos periódicos, tanto nacionales como regionales, que se consagraron al estudio de la carrera política de este dirigente socialista.

En este pequeño trabajo pretendemos, a grandes rasgos, analizar la historiografía que, a lo largo de las últimas décadas, ha estudiado la trayectoria política de Indalecio Prieto, haciendo hincapié en la referente a su acción política durante la Segunda República, período histórico en el que hemos centrado nuestras investigaciones sobre este líder socialista. Con ello trataremos de llegar al estado de la cuestión en el que se encuentra, historiográficamente hablando, la trayectoria política de Prieto durante el período republicano.

\section{EL ESTUDIO DE PRIETO DENTRO DEL CONTEXTO DEL MOVIMIENTO SOCIALISTA ESPAÑOL}

Como apuntamos en la Introducción, durante las décadas de los setenta y los ochenta del siglo XX, sobre todo durante la segunda mitad de los setenta y la primera parte de los ochenta, se publicaron en España numerosas monografías sobre la evolución del movimiento obrero y sobre el devenir de las organizaciones socialistas españolas. Estos trabajos abarcaban principalmente las primeras décadas de vida de las organizaciones obreras en España y la evolución de las mismas durante los años veinte y treinta del siglo XX, por ser esta la etapa en la que mayor auge e influencia tuvieron dentro de la sociedad y de la política españolas.

Es en este contexto historiográfico en el que se analiza la trayectoria política de Indalecio Prieto. Este político fue estudiado como uno de los principales dirigentes y líderes socialistas de las primeras décadas del sigo XX, principalmente durante la

2 Álvarez Junco, J. y PÉRez Ledesma, M. (marzo-abril 1982). "Historia del movimiento obrero. ¿Una segunda ruptura?”. Revista de Occidente. no 12, pp. 19-41; Perez LeDESMA, M. (Domingo 6VI-1982). "Por una nueva ruptura en la historia social". El País. En estos dos artículos sus autores analizan el "boom" historiográfico que se vivió sobre la Historia del movimiento obrero en España durante los años sesenta y setenta, exponiendo las causas de este auge historiográfico y las graves deficiencias metodológicas que, a su juicio, contenían la inmensa mayoría de los estudios que durante esta etapa abordaron la Historia del movimiento obrero español. 
Dictadura de Primo de Rivera, la Segunda República y la Guerra Civil. Estos trabajos estudiaban a Prieto dentro del análisis general de la evolución del PSOE durante el primer tercio del siglo XX.

Por lo tanto, en esta etapa prácticamente no tenemos obras específicas y monográficas sobre Indalecio Prieto, pero el hecho de que este político fuera una de las cabezas más visibles del PSOE de la época y el principal líder de una de las más importantes corrientes ideológicas que convivieron en este partido político, sobre todo durante los años veinte y treinta del siglo pasado, hicieron que Prieto tuviera un lugar destacado y central en las monografías que analizaron la evolución del movimiento socialista español, principalmente en aquellas que se acercaron al estudio de su organización política.

Nuestro objetivo en este apartado es analizar lo que aportan los principales trabajos en los que se ha estudiado a las organizaciones socialistas españolas a la trayectoria política de Prieto durante la Segunda República, centrándonos en aquellas monografías publicadas durante las décadas de los setenta y los ochenta.

Manuel Tuñón de Lara está considerado el punto de referencia esencial en la historiografía contemporánea española de aquellos años. Su ingente actividad se centró en los siglos XIX y XX, estando interesado en la divulgación y en la metodología de la teoría social marxista. Afrontó la Historia Política, Social e Intelectual, siendo los suyos trabajos de grandes dimensiones que inspiraron a la generación de historiadores contemporáneos que se abrió camino desde mediados de los sesenta. Sin embargo, tuvo más admiradores que discípulos, ya que su visión de la Historia del movimiento obrero no tuvo autores capaces de aplicar su metodología con la complejidad que él propugnaba. Su forma de hacer Historia Política basada en el manejo de conceptos políticos tampoco tuvo muchos seguidores a corto plazo. Su concepción de la Historia obrera quedó plasmada en su Historia del movimiento obrero español, en la que se aprecian rasgos de la Historia obrera francesa coetánea. Le daba mucha importancia a las organizaciones, a las que examinaba como representación de manifestaciones de clase y de coyunturas económicas, siguiendo un esquema marxista que le permitiera examinar los rasgos socio-profesionales del mundo obrero. Su obra no fue una Historia obrera tradicional, pero tampoco era una Historia socio-cultural tal y como la entienden las corrientes actuales. Estaría enmarcada dentro de la Historia Económica y Social. Tenía dos concepciones de la Historia social: por una parte tenía la visión annalista de la Historia total y por otra la de la Historia del movimiento obrero como terreno especializado. Se consideraba tributario de la vieja tradición de la Historia obrera y superador de la misma. Era deudor de las tradiciones españolas de la Historia Social y partidario de la Historia Económica y 
Social. Su Historia obrera marca un antes y un después, supuso la fundación de la moderna Historia obrera española y lanzó un desafío a la joven generación de especialistas en Historia Contemporánea de los setenta. Por otra parte, su Historia Política estaba influenciada por el estructuralismo francés de la segunda mitad de los setenta y por su formación en ciencias políticas ${ }^{3}$.

En sus estudios sobre la Segunda República y sobre la evolución del movimiento obrero en España durante los siglos XIX y XX, al abordar el período republicano, aporta como principal novedad sus análisis socio-económicos de la España del momento y la evolución de las organizaciones obreras -para lo que aquí nos interesa, del PSOE- a lo largo de las distintas etapas por las que se deslizó la República, aparte de estudiar las relaciones entre las principales organizaciones sindicales y políticas obreras durante el período republicano. Es dentro de este contexto de Historia general de la República y del movimiento obrero en el que estudia el papel político de Prieto. ${ }^{4}$

Santos Juliá es otro de los historiadores que mejor ha analizado, tanto cualitativa como cuantitativamente, la evolución de las organizaciones socialistas españolas desde su nacimiento hasta los últimos años, centrándose, principalmente, en el devenir de la organización política socialista durante la Segunda República. Por lo tanto, son numerosas las publicaciones en las que analiza la evolución del socialismo español, principalmente durante el período republicano, centrándose en el papel político del PSOE durante esta etapa histórica. ${ }^{5}$ La principal aportación de

${ }^{3}$ PASAMAR, G. op. cit., pp. 239-242. En estas páginas el autor resalta la relevancia de Tuñón de Lara en la historiografía española sobre Historia Contemporánea a partir de los años sesenta, analizando principalmente sus aportaciones a la Historia del movimiento obrero español.

${ }^{4}$ TuÑón De LARA, M. (1985). El movimiento obrero en la Historia de España. Madrid: Sarpe, D.L.; (1976). La Segunda República. 2 vols. Madrid: Siglo XXI. Para la primera obra ver dentro del volumen dedicado al siglo XX la parte que le dedica a la evolución del movimiento obrero durante la República. Hemos incluido aquí la edición de 1985, pero las dos primeras ediciones de esta obra vieron la luz en 1970 y 1972.

5 Algunas de estas obras son: Juliá DíAZ, S. (otoño de 1984). "Continuidad y ruptura en el socialismo español del siglo XX". Leviatán. 2a época. $\mathrm{n}^{\mathrm{o}}$ 17, pp. 121-130; (1983). "Corporativistas obreros y reformadores políticos. Crisis y escisión del PSOE en la Segunda República". Studia Histórica. vol. 1. $\mathrm{n}^{\mathrm{o}}$ 4, pp. 41-52; (1986). El socialismo en España: desde la fundación del PSOE hasta 1975. Madrid: Editorial Pablo Iglesias; (1989). Historia del socialismo español (1931-1939). Barcelona: Conjunto Editorial.; (enero- mayo de 1983). "Los socialistas en la crisis de los años 30". Zona Abierta. $\mathrm{n}^{\circ}$ 27; (1986). "República, revolución y luchas internas". Anales de Historia de la Fundación Pablo Iglesias. vol 1. Madrid.; (1985). "Sindicatos, Partidos y Frente Popular". Leviatán, segunda época. $\mathrm{n}^{\circ}$ 22, pp. 105-112; (julio de 1986). "Sobre la formación del Frente Popular en España”. Sistema. Madrid. no 73. 
los trabajos de Santos Juliá publicados durante estos años deriva de la división que realiza del socialismo español desde su origen en lo que él denomina "corporativistas obreros" (corriente sindical vinculada a la UGT) y "reformadores políticos" (más relacionados con la política y por lo tanto con el PSOE). Para el autor esta dualidad estructural de las organizaciones socialistas en partido político y sindicato estaría en el origen de las graves diferencias ideológicas y tácticas surgidas entre estos dos grupos a lo largo de las décadas de los veinte y de los treinta. Prieto sería el principal líder y portavoz de lo que Juliá denomina "reformadores políticos". Éstos eran partidarios de que la acción política fuera prioritaria frente a la actividad sindical dentro del socialismo español y pretendían que la segunda estuviera subordinada a la primera para que las organizaciones socialistas se integraran e implicaran completamente en el sistema republicano.

Gabriel Jackson en su acreditado manual general sobre la República española y la Guerra Civil que fue publicado en estos años estudia, en la parte que hace referencia a la Segunda República, la evolución general del socialismo durante esta etapa y el papel de Prieto tanto dentro de su propio partido político como en la vida política general de la época. ${ }^{6}$

Por su parte, José Manuel Macarro Vera aporta una novedosa interpretación sobre la radicalización que se llevó a cabo en gran parte de las organizaciones socialistas mediada la República. ${ }^{7}$ El citado autor considera que ésta se produjo, única y casi exclusivamente, con motivo de la salida de los ministros socialistas del Gobierno en septiembre de 1933, desechando el resto de las razones a las que se suele atribuir este proceso de radicalización. En este mismo trabajo hace una serie de alusiones interesantes a Prieto. Subraya de él su papel destacado como firme partidario de mantener la colaboración con los republicanos progresistas y contrario a la implantación de un sistema plenamente socialista en España. Macarro Vera considera que la política de Prieto era la única viable en aquellos momentos dentro del PSOE.

Manuel Contreras también aborda ampliamente la radicalización socialista, aunque desde un punto de vista distinto al de Macarro Vera. ${ }^{8}$ Estamos ante el autor

6 JaCKSON, G. (1999). La República española y la Guerra civil 1931- 1939. Barcelona: Crítica. Aquí incluimos una de las últimas ediciones que han sido publicadas de este manual, la primera de ellas vio la luz en 1976, por esta razón lo hemos incluido en este apartado.

7 Macarro Vera, J. M. (diciembre de 1982). "Causas de la radicalización socialista en la Segunda República”. Revista de Historia Contemporánea. Sevilla. nº 1, pp. 178-224.

${ }^{8}$ CONTRERAS, M. (1987). El PSOE en la Segunda República: organización e ideología. Madrid: Alianza Editorial. 
que más detalladamente analiza la radicalización socialista y que realiza una periodización más clara y precisa de la misma, al menos desde nuestro punto de vista. En cuanto a Prieto, las principales aportaciones de este trabajo son el estudio de su papel en torno a la colaboración con los republicanos durante el primer bienio y el amplio análisis crítico que realiza del Programa de Bases elaborado por el propio Prieto a principios de 1934.

Otras de las principales contribuciones de estos años al conocimiento de la Historia del socialismo español durante la Segunda República son las de Marta Bizcarrondo. ${ }^{9}$ Estos trabajos son muy esclarecedores en varios aspectos: en ellos analiza ampliamente la problemática en torno a las Alianzas Obreras, la política comunista durante la República -sobre todo su relación cambiante con los socialistas- $\mathrm{y}$, principalmente, estudia con detenimiento la división interna socialista acaecida en 1935 y la formación del Frente Popular. Son estos dos últimos aspectos los que más nos interesan para nuestra investigación ya que en ambos procesos Prieto desempeñó un papel determinante y protagonista. La autora reconoce este papel y lo analiza certeramente, pero desde un punto de vista muy crítico hacia él. Esta es, desde nuestro punto de vista, la principal aportación de esta autora al estudio de Prieto. Considera que estaba mucho más próximo a las posiciones políticas e ideológicas de los republicanos progresistas que a las de los socialistas y, además, lo juzga demasiado entreguista hacia los republicanos. Esta autora realiza un análisis muy riguroso y de alta calidad pero claramente favorable a las tesis defendidas en aquellos momentos por el sector socialista liderado por Largo Caballero y desfavorable hacía las amparadas por Prieto.

En esta etapa contamos con algunos autores que estudiaron monográficamente alguna de las corrientes ideológicas que protagonizaron la dinámica interna socialista durante la Segunda República. Es el caso de Andrés de Blas Guerrero y su análisis del sector radical, izquierdista o "caballerista" del socialismo español durante la República. ${ }^{10}$ En este trabajo el autor analiza detalladamente a esta tendencia ideológica socialista desde su gestación y lo hace estudiando su formación, su evolución, sus postulados políticos e ideológicos y a sus principales integrantes. Lo más interesante, para lo que en este trabajo nos concierne, lo

9 Bizcarrondo, M. (enero-junio de 1981). "De las Alianzas Obreras al Frente Popular". Estudios de Historia Social. no 16-17, pp. 83-116; (enero-junio de 1981). "Democracia y revolución en la estrategia socialista en la Segunda República". Estudios de Historia Social. no 16-17, pp. 227-459. Aconsejamos sobre todo este último trabajo, ya que es uno de los más completos que se han publicado hasta el momento sobre la evolución del socialismo español durante el período republicano.

${ }^{10}$ De Blas Guerrero, A. (1978). El socialismo radical en la Segunda República. Madrid: Tucar Ediciones. 
constituye el hecho de que al ir analizando al socialismo "caballerista" acaba adoptando una posición muy crítica hacia el mismo y sobre todo hacia su principal líder y termina realizando un análisis profundo y certero del pensamiento político de Prieto desde un punto de vista favorable hacia el mismo.

En esta etapa tenemos otros estudios que abordan la evolución del socialismo durante la República desde un enfoque regional. Es el caso de Ricardo Miralles y sus trabajos sobre el socialismo vasco ${ }^{11}$. Sin embargo, conviene hacer una importante aclaración al respecto. En nuestra etapa de estudio el socialismo español estaba bastante centralizado organizativamente hablando y, además, desde el socialismo vasco de la época en ningún momento se cuestionó esta centralización. Por lo tanto, las obras de Miralles estudian al socialismo vasco pero a la vez analizan la evolución del socialismo español en general porque el primero estaba fuertemente identificado con el segundo. Una vez hecha esta precisión, podemos señalar que los trabajos de Miralles son fundamentales no sólo para conocer al socialismo vasco y español de la época sino que son imprescindibles para el estudio de Prieto durante la República ya que el socialismo vasco (su inmensa mayoría) estuvo fuertemente identificado en esta etapa con las posiciones políticas de su líder. El autor analiza fundamentalmente el papel de Prieto en el advenimiento de la República, su postura ante la radicalización y, sobre todo, su papel después de Octubre de 1934 en la división interna del PSOE y en la formación del Frente Popular.

En último lugar vamos a hacer referencia a un trabajo en el que Manuel Redero San Román realiza una recensión de algunas de las principales obras publicadas en esta etapa sobre la evolución del socialismo español durante la República. En este trabajo el autor lleva a cabo una síntesis de cada una de estas obras destacando las principales aportaciones de cada una de ellas al estudio del movimiento socialista durante el período republicano. Algunos de los trabajos que analiza este autor han sido mencionados en este epígrafe. ${ }^{12}$

11 Miralles, R. (1988). "El particularismo político del socialismo vasco". Historia Contemporánea. UPV. ${ }^{\circ} 1$, pp. 109-122; (1988). El socialismo vasco durante la Segunda República. Bilbao: Universidad del País Vasco.

12 Redero SAn Román, M. (1983). "El movimiento socialista de la Segunda República en la historiografía de su cincuentenario". Studia Histórica, Historia Contemporánea. vol. 1. no 4, pp. 175179. En este trabajo el autor analiza varias de las obras más destacadas de Marta Bizcarrondo, Santos Juliá y Manuel Contreras. Todas ellas fueron publicadas durante el trienio comprendido entre 1981 y 1983. 


\section{APOGEO DE LOS ESTUDIOS MONOGRÁFICOS SOBRE PRIETO}

A comienzos de los ochenta, una vez culminada la transición a la democracia, se produjo un punto de inflexión en el desarrollo de los estudios de Historia Contemporánea en España.

Algunos autores como Álvarez Junco y Pérez Ledesma reclamaban una Historia del movimiento obrero menos influida por la militancia política, más apoyada en aquellas ciencias sociales más próximas a la Historia como la sociología, la politología, la antropología o la psicología social, ampliando el campo de estudio y multiplicando los enfoques para incluir la Historia del movimiento obrero dentro del campo más amplio de los movimientos sociales. ${ }^{13}$.

Durante estos años tenemos varios signos de asentamiento de la Historia del movimiento obrero, relativamente retrasado en España por la censura franquista. Para algunos autores, como los citados anteriormente, la movilización antifranquista fue la que hizo que se aplazara una auténtica renovación metodológica en favor de los objetivos políticos, mientras que otros consideran que la militancia política no limitó la capacidad investigadora de los historiadores.

Durante los años ochenta se originó un auge de la historiografía española en general, sobre todo de la Historia Contemporánea. Se dio un interés creciente por la Historia del Tiempo Presente, una renovación de la Historia Política -más en contacto con la politología y con la sociología electoral-, una influencia de la nueva Historia Económica, una proliferación de la Historia Regional y Local y nuevas formas de hacer Historia Social como la Historia de Género, de la Vida Cotidiana y de las Mentalidades. En cuanto a la Historia Política se llevó a cabo un proceso de renovación en los estudios sobre la Restauración, el Franquismo y la Transición, con influencia de la historiografía francesa, de la Historia del Tiempo Presente y un mayor contacto con las ciencias sociales.

13 Perez Ledesma, M. op. cit.; Álvarez Junco, J. y Pérez Ledesma, M. op. cit., pp. 19-41. Como ya apuntamos en la Introducción estos dos autores realizan una crítica general bastante severa de los numerosos estudios que sobre el movimiento obrero se realizaron en España durante los años sesenta y setenta. Consideran que estos trabajos fueron positivos y saludables en el contexto de la Dictadura de Franco, ya que suponían realizar la Historia de los vencidos en la Guerra Civil, pero estaban muy influenciados por la lucha política contra el franquismo. Según estos autores esta nueva disciplina historiográfica no supuso una renovación metodológica de la manera de construir el relato histórico, sino más bien todo lo contrario, ya que detectan graves deficiencias metodológicas en estos trabajos, muchas de ellas heredadas de la manera tradicional de hacer Historia, a la que, en teoría, pretendían superar. Proponían que la Historia del movimiento obrero se despolitizara y se integrara como una disciplina más en el mundo académico. 
Durante los años ochenta aumentó considerablemente el número de revistas especializadas sobre Historia Económica y Social y sobre Historia Contemporánea, tendencia que continuó a lo largo de los noventa.

En el marco de éste auge historiográfico, los primeros años de la década de los ochenta, sobre todo 1983, vivieron un apogeo de los estudios monográficos sobre Prieto. Hasta este momento, como hemos comprobado en el epígrafe anterior, las disertaciones sobre Prieto estaban circunscritas a su papel político dentro de las organizaciones socialistas en las que militaba desde su juventud. A partir de ahora se le va a analizar individualmente, se realizan investigaciones de conjunto de su carrera política y, sobre todo, se llevan a cabo estudios concretos sobre algunas de sus más destacadas actuaciones políticas, la mayoría de ellas llevadas a cabo durante la Segunda República.

En general, las publicaciones que sobre Prieto vieron la luz durante los primeros años de la década de los ochenta fueron artículos divulgados a través de la prensa de la época -tanto nacional como regional- y artículos dados a conocer en el marco de revistas especializadas sobre Historia española contemporánea o sobre ciencias políticas.

Destaca la cantidad de monografías que se publicaron sobre Prieto en 1983. La razón de este auge bibliográfico se debe a que durante ese año se conmemoró el centenario de su nacimiento. Por este motivo se publicaron en periódicos y en revistas especializadas numerosas reseñas sobre su figura política.

Dentro de las publicaciones que sobre Prieto vieron la luz en el año del centenario de su nacimiento, una de las más destacables es el número especial y casi monográfico que le dedicó la Revista del Ministerio de Obras Públicas y Urbanismo en su edición de diciembre de $1983 .{ }^{14}$ Este número especial de la revista del MOPU incluye una pequeña autobiografía escrita en 1930 y siete artículos en los que algunos de los más destacados historiadores, politólogos y colaboradores de Prieto destacan varias de las facetas más importantes de su vida política, la mayoría de ellas llevadas a cabo durante la Segunda República. A continuación analizaremos lo que aportan estos artículos al estudio de Prieto.

En primer lugar y como prólogo al homenaje, el socialista Julián Campo -que en aquellos momentos era Ministro de Obras Públicas en el primer Gobierno de Felipe

\footnotetext{
${ }^{14}$ Conviene recordar que Prieto desempeñó el cargo de ministro de Obras Públicas durante casi dos años a lo largo del primer bienio republicano y que su gestión al frente de este Ministerio ha sido valorada casi unánimemente como positiva por la comunidad científica.
} 
González-, lleva a cabo una valoración general de la gestión de Prieto al frente de este mismo Ministerio ${ }^{15}$. En ella destaca la trascendencia de esta gestión y las virtudes políticas que, -a su juicio- poseía Prieto. Considera que su labor en Obras Públicas estuvo presidida por la combinación de varios factores que le dieron trascendencia de hombre de Estado ya que intentó racionalizar los recursos, atender las demandas sociales de la época y dinamizar la situación económica a través de la inversión del Estado en Obras Públicas para paliar el paro y minimizar los desequilibrios regionales. Para Julián Campo la relevancia de Prieto en Obras Públicas radica de la visión de futuro de sus proyectos y de su intento por enmarcarlos dentro de una política general regeneracionista auspiciada por el Gobierno progresista del que formaba parte. Este artículo constituye una aportación interesante para el conocimiento de la gestión de Prieto en Obras Públicas, en el lado negativo situamos lo limitado del espacio y la visión subjetiva del autor hacia el personaje analizado.

El periodista Jorge Martínez Reverte realiza una curiosa aportación a este homenaje a Prieto ${ }^{16}$. En un pequeño artículo escrito con un lenguaje muy claro y rotundo, destaca el papel político de Prieto desde Octubre de 1934 hasta la Guerra Civil. Lo reivindica considerándolo como uno de los pocos dirigentes políticos presentables de toda la Historia del socialismo español. Subraya su figura de socialista moderado, demócrata y responsable frente a Besteiro y a Largo Caballero ante los que se muestra muy crítico, sobre todo con éste último. Considera que la debilidad y el moderantismo de Besteiro y el radicalismo y la irresponsabilidad de Largo Caballero fueron muy perjudiciales tanto para el PSOE como para la República. Por ello reivindica la figura de Prieto, desde un punto de vista apasionado y subjetivo pero con un lenguaje muy esclarecedor.

Santos Juliá, por su parte, lleva a cabo una semblanza general de Prieto en la que recalca que fue el líder más destacado de la corriente política existente dentro del PSOE, principalmente durante las décadas de los veinte y de los treinta. Esta tendencia política auspiciada por Prieto abogaba por la colaboración socialista con los republicanos progresistas para consolidar en España un sistema democrático con alto contenido social que mejorara sustancialmente las condiciones de trabajo y de vida de la clase trabajadora. Para Juliá la dualidad estructural del socialismo español en organización política y sindical está en la base la división interna

${ }^{15}$ CAMPO, J. (diciembre de 1983). "La sombra alargada de Indalecio Prieto". Revista del MOPU. $\mathrm{n}^{\mathrm{o}} 305, \mathrm{p} .5$.

${ }^{16}$ Martínez Reverte, J. (diciembre de 1983). "Un panfleto prietista". Revista del MOPU. $\mathrm{n}^{\mathrm{o}}$ 305, pp.16-17. 
socialista durante esta etapa, siendo Prieto el principal portavoz del sector político del PSOE. ${ }^{17}$

Paul Preston ${ }^{18}$ analiza el papel político de Prieto desde la salida de los ministros socialistas del Gobierno en septiembre de 1933 hasta las negociaciones que desembocaron en la firma del pacto del Frente Popular, todo ello desde un punto de vista bastante favorable. Sin embargo, su artículo, aunque estudia a Prieto principalmente en la etapa que hemos apuntado anteriormente, acaba haciendo una semblanza general de su trayectoria política y de la evolución de las organizaciones socialistas durante la Segunda República.

Por su parte, José Prat, que fue compañero de partido, coetáneo y colaborador de Prieto durante la etapa de la Guerra Civil realiza una semblanza general, cariñosa y entrañable de Prieto ${ }^{19}$. Incide, ante todo, en su destacado papel en contra de la Guerra de Marruecos, en su gestión al frente de los Ministerios de Hacienda y de Obras Públicas y en su actividad política durante su exilio final en México. Es una aportación interesante $\mathrm{y}$ un testimonio de primera mano, aunque hay que analizarlo teniendo en cuenta la admiración que Prat profesaba a Prieto y la amistad que unió a ambos.

Manuel Díaz Marta -que fue colaborador de Prieto durante su etapa de ministro de Obras Públicas,- analiza en un artículo monográfico lo más destacado de su gestión al frente del citado Ministerio ${ }^{20}$. Es un análisis muy completo e imprescindible para conocer a fondo la labor de Prieto en Obras Públicas, sobre todo para examinar su fructífera política de obras hidráulicas. Además realiza una semblanza general en la que destaca las virtudes políticas y personales de Prieto. También está realizado desde un punto de vista favorable aunque es un estudio

17 Juliá DíAZ, S. (diciembre 1983). "Un líder político entre dirigentes sindicales". Revista del MOPU. $n^{\circ} 305$, pp. 26-30; (primavera de 1974). "La vigencia de Prieto“. Leviatán, $2^{\mathrm{a}}$ época. $\mathrm{n}^{\mathrm{o}} 15$, pp. 71-76. Este último artículo también analiza la figura política de Prieto destacando sus virtudes y defectos políticos. En este caso destaca el hecho de que esta publicación esté datada en 1974, por lo que puede ser considerada precursora de los estudios individualizados que se fueron publicando más adelante sobre Prieto.

18 Preston, P. (diciembre de 1983) ."Demócrata por encima de todo: Indalecio Prieto y la creación del Frente Popular". Revista del MOPU. n 305, pp. 37-41.

19 PrAT, J. (diciembre de 1983). "Acción y pasión en Indalecio Prieto". Revista del MOPU. $\mathrm{n}^{\circ}$ 305, pp. 31-36.

${ }^{20}$ DíAz MARTA, M. (diciembre de 1983). "Indalecio Prieto en Obras Públicas: una gestión intensa y acertada". Revista del MOPU. n 305, pp. 11-15. 
muy riguroso sobre su labor desempeñada al frente del Ministerio de Obras Públicas.

Juan Pablo Fusi ha analizado en múltiples obras tanto la evolución del movimiento obrero como la del nacionalismo en el País Vasco. También ha estudiado, en relación con estos dos aspectos, la actitud de Prieto ante la cuestión vasca en general y sobre el Estatuto de autonomía para el País Vasco durante la Segunda República en particular ${ }^{21}$. La principal tesis de Fusi reside en que Prieto y los socialistas vascos estaban muy enfrentados políticamente con el PNV por lo dispar de sus proyectos políticos y de las fuerzas sociales a las que representaban. Los socialistas vascos pretendían un régimen de autonomía para el País Vasco dentro de una España democrática, constitucional y laica mientras que los nacionalistas vascos estaban caracterizados por su fuerte nacionalismo, su clericalismo y su conservadurismo. Destaca que Prieto como principal y casi indiscutido líder del socialismo vasco durante los años treinta, rechazó el proyecto autonomista auspiciado por el PNV en los primeros años republicanos por sus connotaciones nacionalistas y conservadoras. En cambio, fue el principal artífice de la asunción por parte del Frente Popular de la política autonomista para el País Vasco y desempeñó un papel determinante en la elaboración del Estatuto en los meses centrales de 1936. Las aportaciones de Fusi son fundamentales para conocer el papel y la postura de Prieto ante la cuestión vasca, sobre todo durante el período republicano.

Por otra parte, la principal aportación al estudio de la gestión de Prieto en el Ministerio de Hacienda durante los primeros meses de vida de la Segunda República la realizó Juan Velarde ${ }^{22}$. Este autor realiza un análisis profundo y técnico de las principales medidas llevadas a cabo por Prieto en este Ministerio, aunque sin desviarse de los aspectos políticos. Su principal tesis reside en que Prieto, aunque era una persona cauta y la situación financiera era mala, actuó con firmeza y de forma progresista en el Ministerio de Hacienda, lo que hizo que fuera sustituido en el cargo por presiones de la oligarquía financiera. Es un análisis muy favorable a Prieto pero a la vez es un estudio riguroso y fundamental para conocer su gestión en Hacienda y las razones de su salida de este Ministerio en diciembre de 1931.

${ }^{21}$ Fusi AizPurua, J. P. (1978). "Indalecio Prieto y el Estatuto vasco de las izquierdas". Homenaje a Julio Caro Baroja. Madrid, pp. 391-403; (diciembre de 1983). "Prieto y el problema vasco". Revista del MOPU. $\mathrm{n}^{\mathrm{o}} 305$, pp. 18-25.

22 Velarde Fuentes, J. (1983). "Indalecio Prieto en Hacienda". Studia Histórica, Historia Contemporánea. vol. 1. n $\mathrm{n}^{\mathrm{O}}$, pp. 53-66. 
Con motivo de la conmemoración del centenario del nacimiento de Prieto se publicaron en la prensa una serie de artículos que reseñaban a éste dirigente socialista. Destacaremos algunos de los que fueron publicados en diarios nacionales, aunque también vieron la luz algunos artículos sobre Prieto divulgados a través de periódicos de ámbito regional, fundamentalmente en el País Vasco ${ }^{23}$. La edición del diario El País de uno de mayo de 1983 publicó dos artículos sobre él, uno del historiador Ian Gibson y otro del profesor de Derecho Internacional Miguel Ángel Marín. En el primero de ellos Gibson realiza una valoración general de conjunto y, desde un punto de vista positivo, de la trayectoria política de Prieto. Es una síntesis general muy interesante y que además hace referencia al papel desempeñado por este político durante la Segunda República, por lo que resulta útil para nuestro ámbito de investigación.

Por su parte, Marín hace referencia a la vida de Prieto hasta 1930 y a algunas de las más importantes gestiones que llevó a cabo en su exilio final mexicano en favor de los exiliados españoles. En este caso no aporta nada sobre la trayectoria política de Prieto durante el período republicano.

La edición del diario A.B.C. de 28 de diciembre de 1983 incluía un pequeño artículo del periodista Lorenzo López Sancho en el que hacía una pequeña semblanza de Prieto. En el mismo se reivindica su figura política y se le elogia como político y como persona, pero sin apenas dar argumentos. El autor muestra su admiración hacia Prieto pero contribuye muy poco a su estudio.

Como hemos podido apreciar, los estudios monográficos que sobre Prieto fueron publicados en los primeros años de la década de los ochenta -sobre todo en 1983- aportan dos novedades fundamentales. En primer lugar era la primera vez que proliferaban los estudios individualizados sobre este líder político y, en segundo término, abordaban el estudio de sus actuaciones políticas más destacadas durante la Segunda República, llenando, en gran parte, el vacío historiográfico existente hasta aquellos momentos sobre estas facetas de su carrera política.

23 Algunos de estos artículos son: GIBSON, I. (1-V-1983). "Socialista pragmático" (sobre Prieto). El País, p.18; LóPez SANChO, L. (28-XII-1983). "Indalecio Prieto". A.B.C., p.18; MARÍn, M. A. (1V-1983). "Una biografía incompleta" (Prieto). El País, p.18; MúgiCa Herzog, E. (1-III-1984). "Prieto y el nacionalismo". El Correo español- El Pueblo vasco.; Olabarri Gortazar, I. (9- V1983). "Indalecio Prieto: el fracaso de un gran político". El Diario Vasco. 


\section{ULTIMAS APORTACIONES HISTORIOGRÁFICAS AL ESTUDIO DE PRIETO}

Algunos autores como Gonzalo Pasamar al analizar la situación de la historiografía española al finalizar el siglo XX llegan a una serie de conclusiones. Entre ellas está el hecho de que desde los años noventa ha aumentado la sociabilidad de los historiadores españoles aunque la diversificación $\mathrm{y}$ compartimentación de la Historia Contemporánea no alcanza a la de las historiografías de los países de nuestro entorno. Los historiadores españoles han roto su tradicional penuria asociativa y tienen conocimiento de las novedades de la historiografía internacional, ya que todas las corrientes historiográficas de trascendencia mundial tienen algunos seguidores en España. La historiografía española habría agotado en algo más de una década lo que otras historiografías han desarrollado en varias, sin embargo, todavía no ha alcanzado un nivel internacional.

Las principales insuficiencias que se detectan en la historiografía española de los últimos años son las siguientes: exigua capacidad de participación en los debates teóricos internacionales, escasez de investigadores que no sólo se dediquen a la Historia de ámbito nacional, un sistema universitario que multiplica la dispersión, un escaso impulso hacia la construcción de síntesis y hacia la elaboración teórica y una excesiva autocomplacencia del gremio de historiadores españoles. La historiografía española actual no carece de información ni de medios de investigación, pero adolece de una tradición de reflexión teórica y de un interés por la Historia internacional que le permita acceder plenamente a los foros mundiales. ${ }^{24}$

En el marco de este contexto historiográfico, durante la última década del siglo $\mathrm{XX}$ y los primeros años del nuevo milenio, la cantidad de trabajos que han sido publicados sobre Prieto y sobre su papel dentro de la dinámica de las organizaciones socialistas ha disminuido considerablemente. Sin embargo, durante todo este tiempo no han dejado de publicarse estudios referentes a estas dos cuestiones. Incluso en los últimos años, debido al auge que han experimentado las biografías históricas han salido a la luz algunas semblanzas muy interesantes sobre Prieto $^{25}$.

${ }^{24}$ Pasamar, G. op. cit., pp. 246-248; MoRadiellos, E. (1992). Las caras de Clio. Introducción a la Historia y a la Historiografia. Oviedo: Universidad de Oviedo. Observar las referencias que estos autores realizan sobre la historiografía española de estos años.

25 Sobre el auge de la biografía histórica en los últimos diez o quince años ver: GómEZNAVArRo, J. L. (abril de 2005). "En torno a la biografía histórica”. Historia y política. $\mathrm{n}^{\circ}$ 13, pp. 7- 
Se suele considerar que la Historia del movimiento obrero y la de sus principales líderes políticos es un género historiográfico que tuvo su máximo apogeo en décadas anteriores y que ya no despierta el interés ni de la comunidad científica ni del público en general. Sin embargo, el número de publicaciones que sobre este campo se han realizado en los últimos quince años desmiente esta creencia muy extendida entre la comunidad de historiadores.

Algunos de los autores que más han aportado, tanto cuantitativamente como cualitativamente, al estudio del movimiento obrero español -sobre todo al movimiento socialista- y al análisis de algunos de los principales líderes obreros, han seguido publicando trabajos sobre este género en los últimos años. Aunque en muchos casos estas publicaciones son versiones aumentadas, revisadas y perfeccionadas de sus estudios anteriores siguen manteniendo una calidad científica notable debido al avance historiográfico que sobre estas materias se ha producido en las últimas décadas. Estos autores son principalmente Manuel Tuñón de Lara, Santos Juliá y Paul Preston.

El profesor Tuñón de Lara continuó divulgando, en sus últimos años de vida, trabajos sobre la dinámica político-social durante la Dictadura de Primo de Rivera y la Segunda República. En ellos analiza la evolución de los principales partidos políticos y sindicatos durante esos años de una manera profunda y certera, incidiendo en la relación entre partidos políticos, ideología y movimientos sociales y en las graves deficiencias y problemas que tuvo el sistema de partidos durante la Segunda República, sobre todo durante sus primeros años de vida. ${ }^{26}$

Santos Juliá, que está considerado como uno de los más notables especialistas sobre la Historia del socialismo español, también ha publicado en los últimos años algunas obras que vienen a completar sus estudios anteriores sobre la dinámica política durante la Segunda República y acerca del PSOE. ${ }^{27}$ Estas obras analizan de

26. En este trabajo el autor realiza una interesante reflexión sobre las razones que provocaron que este género historiográfico pasara a un segundo plano desde el final de la Segunda Guerra Mundial hasta los años setenta y que en la actualidad esté en auge. También analiza los principales tipos de biografías históricas y los problemas que pueden surgirle al historiador que se acerque a este género historiográfico.

26 Ver Tuñón De LARA, M. (1991). “El sistema de partidos en 1931-1933”. Historia Contemporánea. UPV. no 6, pp. 59-84 y (1990). "Transformaciones políticas e ideológicas de España durante el primer tercio del siglo XX”. Historia Contemporánea. UPV. n 4 , pp. 231-258.

${ }^{27}$ Ver Julí́ DíAz, S. (1996). "El sistema de partidos en la Segunda República”. Revista Historia Contemporánea. Sevilla. no 7, pp. 201-218; (2003). La España del siglo XX. Madrid: Marcial Pons Historia; (1997). Los socialistas en la política española, 1879-1982. Madrid: Taurus. De estas dos 
manera completa, clara y certera tanto el complejo sistema de partidos de la Segunda República con sus divisiones internas y sus escisiones, como las luchas intrínsecas dentro del PSOE durante esa etapa histórica. Para lo que aquí nos interesa, analiza ampliamente tanto el papel protagonista de Prieto dentro de la convulsa dinámica interna socialista durante el período republicano como su gestión al frente de los Ministerios de Hacienda y de Obras Públicas. Estas tres obras vienen a completar las investigaciones que ha realizado este autor tanto sobre los partidos políticos de la Segunda República como acerca del PSOE.

Paul Preston también ha seguido publicando durante estos años diversos estudios sobre la dinámica del socialismo español durante los años treinta y acerca de la carrera política de Prieto. ${ }^{28}$ Sobre la primera cuestión Preston considera que la división interna socialista fue una de las razones que provocaron la caída de la República y que la radicalización de las organizaciones socialistas durante el período republicano se debió a la presión de los jornaleros del sur y de los mineros del norte que estaban afiliados a la UGT a través de la Federación Nacional de Trabajadores de la Tierra (FNTT) y del Sindicato Obrero Minero de Asturias (SOMA).

Pero lo que más nos concierne para nuestra investigación es la pequeña biografía que sobre Prieto incluye en Las tres Españas del 36. En ella ofrece una visión general pero completa tanto de la vida personal de Prieto desde su nacimiento hasta su muerte como de toda su carrera política. La principal novedad de esta biografía radica en que aporta un interesante análisis de los principales rasgos de carácter de Prieto y como éstos influyeron en su carrera política. Esto es muy importante ya que Prieto era una persona con un carácter muy complejo y cambiante que determinó, en gran medida, muchas de sus actuaciones políticas.

Como podemos apreciar, durante esta etapa hay una combinación de trabajos que estudian a Prieto individualmente y otros que abordan la trayectoria política de este líder socialista dentro del contexto del movimiento socialista español. Entre este último tipo de obras tenemos dos aportaciones interesantes de sendos historiadores que han abordado en múltiples estudios la evolución del socialismo español, una es de Manuel Redero San Román y la otra de Francisco de Luis

últimas obras y para lo que a nuestra investigación interesa ver los capítulos referentes a la Segunda República y a la Guerra Civil.

${ }^{28}$ Ver Preston, P. (1998). Las tres Españas del 36. Indalecio Prieto, una vida a la deriva. Barcelona: Plaza y Janés y (2001). La Destrucción de la democracia en España. Barcelona: Grijalbo Mondadori. Para esta última obra conviene analizar especialmente los capítulos dedicados a la evolución del PSOE-UGT durante la Dictadura de Primo de Rivera y la Segunda República. 
Martín ${ }^{29}$. El primero de ellos realiza una síntesis bastante completa de la evolución conjunta del socialismo español -PSOE y UGT- durante la Segunda República, haciendo hincapié en las divisiones internas y con un análisis muy completo de las distintas razones que provocaron la radicalización de una parte sustancial de las organizaciones socialistas desde 1933 en adelante.

Por su parte, Francisco de Luis Martín amplía el estudio de la evolución del socialismo español a la etapa de la Dictadura de Primo de Rivera. Es un trabajo que proporciona al lector una explicación muy clara y ágil sobre la acción socialista durante estos años, con sus divisiones internas, y sobre las posiciones políticas de Prieto. Destaca que este político socialista lideró la tendencia socialdemócrata del PSOE y mantuvo, durante esta etapa, la coherencia de sus postulados políticos: Estado democrático con alto contenido social en el que se integrara plenamente el socialismo.

La principal aportación de estas dos obras, al margen de las numerosas e interesantes alusiones que ambos autores hacen a Prieto, lo constituye el hecho de que realizan una síntesis de la evolución del socialismo español durante esa etapa a través de las investigaciones propias y de una recapitulación de la historiografía existente sobre la cuestión hasta aquellos momentos.

Como hemos visto precedentemente, la figura política de Prieto no escapó al auge que han experimentado las biografías históricas en los últimos años y tenemos varios estudios biográficos publicados en los últimos tiempos sobre él. El más completo, junto con la biografía de Preston en Las Tres Españas del 36, es la que le dedica Octavio Cabezas. ${ }^{30}$ Esta es, desde nuestro punto de vista, la obra más amplia y completa de todo lo que se ha escrito hasta estos momentos sobre Prieto. Es una biografía eminentemente política, aunque no desprecia los aspectos personales (sobre todo, como es lógico, cuando aborda sus primeros años de vida y su adolescencia), en la que abarca toda su vida. Estamos ante una semblanza extensa, integral, que está muy bien redactada y documentada, ya que, sobre todo en la parte alusiva a la Guerra Civil, aporta documentación inédita que le ha sido facilitada al autor por parte de la Fundación Indalecio Prieto. La principal aportación de esta obra, aparte de la complejidad del estudio, es la combinación que realiza el autor entre el contexto histórico y la acción política concreta de Prieto. Sobre su labor

${ }^{29}$ Estos dos trabajos son; DE LuIS MARTín, F. (1998). "Consideraciones sobre las relaciones Socialismo- Estado en España (1879-1936)”. Historia Contemporánea. UPV. no 17, pp. 309-333 y; REDero SAn RomÁn, M. (1992). Estudios de Historia de la UGT. El movimiento socialista en la Segunda República (1931-1936). Salamanca: Ediciones Universidad de Salamanca.

${ }^{30}$ CabeZas, O. (2005). Indalecio Prieto, socialista y español. Madrid: Algaba Ediciones. 
durante el período republicano analiza ampliamente tanto su gestión en los Ministerios de Hacienda y de Obras Públicas, como sus posiciones en torno a la cuestión de la autonomía vasca, así como su postura ante la revolución de Octubre de 1934, aparte de examinar su destacado papel dentro del PSOE durante aquellos años.

Esta obra es, desde nuestro punto de vista, lo más amplio, completo y mejor documentado de todo lo que se ha publicado hasta estos momentos sobre Prieto.

\section{ALCANCES Y LÍMITES DE LA PRODUCCIÓN HISTORIOGRÁ- FICA EXISTENTE SOBRE PRIETO}

Como conclusión a este pequeño estudio sobre la situación historiográfica en la que se encuentra la trayectoria política de Indalecio Prieto durante la Segunda República, podemos afirmar que se ha estudiado ampliamente su papel dentro de la convulsa dinámica interna socialista, sobre todo durante las décadas de los veinte y treinta. En el marco del auge de los estudios históricos sobre el movimiento obrero y acerca del movimiento socialista español acaecido durante los años setenta y ochenta, se estudió el papel protagonista de Prieto dentro del PSOE. Éste fue el principal líder de la corriente centrista o socialdemócrata dentro del Partido Socialista y como tal fue uno de los principales protagonistas de la lucha interna que se produjo entre las distintas sensibilidades ideológicas que convivieron dentro de esta organización política durante estos años, en particular durante la Segunda República, etapa en la que Prieto emergió más que nunca como uno de los políticos más destacados de aquella época. Por lo tanto, las obras que estudiaron el desarrollo del movimiento socialista español de los años republicanos aportan al conocimiento de Prieto el análisis de su destacado papel dentro de la dinámica interna socialista.

No será hasta los primeros años de la década de los ochenta cuando se estudie a Prieto de manera individualizada. Como hemos podido apreciar, durante esos años y al calor del auge de los estudios sobre Historia Contemporánea en España, se publicaron múltiples monografías que abordaban su carrera política íntegramente y otras que afrontaban algunas de sus principales actividades políticas durante el período republicano: especialmente su gestión en los Ministerios de Hacienda y de Obras Públicas y su postura acerca de la autonomía vasca. Todos estos trabajos venían a ampliar, completar e individualizar los estudios historiográficos que hasta aquellos momentos se habían realizado sobre Prieto.

Durante la década de los noventa y a lo largo de los primeros años del nuevo siglo, la producción historiográfica sobre Prieto y la relativa al socialismo español bajó notablemente, sin embargo, durante todos estos años no han dejado de 
publicarse trabajos sobre estas dos cuestiones. Esto demuestra que son materias que siguen interesando tanto a la comunidad científica como al público en general. Algunos de los trabajos que han sido publicados sobre Prieto en los últimos tiempos se han realizado al calor del auge de los estudios biográficos y demuestran que sigue siendo uno de los personajes históricos contemporáneos españoles que mayor interés sigue despertando.

Lo más importante de estas últimas aportaciones lo constituye el hecho de que están realizadas -tanto las referentes a Prieto como al socialismo-, desde una doble vertiente que los hace mucho más completos. Por una parte se basan en las contribuciones de la numerosa historiografía existente sobre estas dos cuestiones y, por otra parte, aportan su propia interpretación de las fuentes y de la bibliografía.

Sin embargo, aunque la trayectoria política de Prieto durante la Segunda República y su carrera política en general ha sido muy estudiada por los científicos sociales, de manera certera y cualificada (en la mayoría de los casos), creemos que hay una faceta que sobre él ha sido muy poco o nada abordada por la historiografía. Es la de su relación con los partidos republicanos progresistas, principalmente con sus líderes, ya que se ha destacado el interés de Prieto por llegar a acuerdos con ellos pero no se ha estudiado en profundidad su relación con sus principales dirigentes, salvo algunas alusiones a su trato político con Azaña (sobre todo durante las negociaciones que desembocaron en la firma del pacto del Frente Popular) y a su amistad con Marcelino Domingo y con Felipe Sánchez Román. Quizá sea esta la única faceta política de Prieto durante la República que no ha sido estudiada con la intensidad que sería deseable.

\section{BIBLIOGRAFÍA UTILIZADA:}

Álvarez Junco, J. y Pérez Ledesma, M. (marzo-abril 1982). "Historia del movimiento obrero. ¿Una segunda ruptura?". Revista de Occidente. $\mathrm{n}^{\mathrm{o}} 12$.

BizCARrondo, M. (enero- junio de 1981). "De las Alianzas Obreras al Frente Popular". Estudios de Historia Social. no 16-17.

BIZCARRONDO, M. (enero- junio de 1981). "Democracia y revolución en la estrategia socialista en la Segunda República". Estudios de Historia Social. ${ }^{\circ}$ 16-17.

BIZCARRONDO, M. (1973). "La crisis socialista en la Segunda República". Revista del Instituto de Ciencias Sociales. $\mathrm{n}^{\circ} 21$. Barcelona.

BIZCARRONDO, M. (1986). "La Segunda República: ideologías socialistas". El socialismo en España. Anales de Historia de la Fundación Pablo Iglesias. vol. 1. Madrid.

BIZCARRONDO, M. "Socialistas y comunistas ante la unidad. Las Alianzas Obreras 
en 1935”. Estudios sobre Historia de España. Homenaje a Manuel Tuñón de Lara. U.I.M.P. Santander.

CABEzAS, O. (2005). Indalecio Prieto, socialista y español. Madrid: Algaba Ediciones.

CAMPO, J. (diciembre de 1983). "La sombra alargada de Indalecio Prieto". Revista del MOPU. $\mathrm{n}^{\circ} 305$.

CONTRERAS, M. (1987). El PSOE en la Segunda República: organización e ideología. Madrid: Alianza Editorial.

De Blas Guerrero, A. (1978). El socialismo radical en la Segunda República. Madrid: Tucar Ediciones.

DE LUIS MARTíN, F. (1998). "Consideraciones sobre las relaciones SocialismoEstado en España (1879-1936)". Historia Contemporánea. UPV. n 17.

DíAZ MARTA, M. (diciembre de 1983). "Indalecio Prieto en Obras Públicas: una gestión intensa y acertada". Revista del MOPU. n 305.

FUSI AIZPURUA, J. P. (1978). "Indalecio Prieto y el Estatuto vasco de las izquierdas". Homenaje a Julio Caro Baroja. Madrid.

FUSI AIZPURUA (diciembre de 1983). "Prieto y el problema vasco". Revista del MOPU. $\mathrm{n}^{\circ} 305$.

García PÉREz BAnCeS, J. (1983). Indalecio Prieto Tuero, 1883-1962. Oviedo: Imprenta Gofer.

GiBAJA VelázQuez, J. C. (1995). Indalecio Prieto y el socialismo español. Madrid: Editorial Pablo Iglesias.

GIBSON, I. (1-V-1983). "Socialista pragmático" (sobre Prieto). El País.

GÓMEZ- NAVARRO, J. L. (abril de 2005). "En torno a la biografía histórica". Historia y política. $\mathrm{n}^{\mathrm{o}} 13$.

IGGERS, G. (1998). La ciencia histórica en el siglo XX. Las tendencias actuales. Una visión panorámica crítica del debate internacional. Barcelona: Idea Universitaria.

JACKSON, G. (1999). La República española y la Guerra civil 1931- 1939. Barcelona: Crítica.

JULIÁ DÍAZ, S. (otoño de 1984). "Continuidad y ruptura en el socialismo español del siglo XX”. Leviatán. $2^{\mathrm{a}}$ época. $\mathrm{n}^{\mathrm{o}} 17$.

JULIÁ DÍAZ, S. (1983). "Corporativistas obreros y reformadores políticos. Crisis y escisión del PSOE en la Segunda República”. Studia Histórica. vol. 1. nº 4.

Juliá DíAZ, S. (1996). "El sistema de partidos en la Segunda República". Revista Historia Contemporánea. Sevilla. $\mathrm{n}^{\mathrm{0}} 7$.

JULIÁ DÍAZ, S. 1986). El socialismo en España: desde la fundación del PSOE hasta 1975. Madrid: Editorial Pablo Iglesias.

Juliá DíAZ, S. (1989). Historia del socialismo español (1931-1939). Barcelona: Conjunto Editorial. 
JULiÁ DíAZ, S. (2003). La España del siglo XX. Madrid: Marcial Pons Historia. JULIÁ DÍAZ, S. (1977). La izquierda del PSOE (1935-1936). Madrid: Siglo XXI. JuliÁ DíAZ, S. (primavera de 1974). "La vigencia de Prieto". Leviatán. $2^{\mathrm{a}}$ época. $\mathrm{n}^{\mathrm{o}}$ 15.

Juliá DíAZ, S. (enero- mayo de 1983). "Los socialistas en la crisis de los años 30". Zona Abierta. $\mathrm{n}^{\circ} 27$.

JULIÁ DíAZ, S. (1997). Los socialistas en la política española, 1879-1982. Madrid: Taurus.

Juliá DíAZ, S. (1979).Orígenes del Frente Popular en España (1934- 1936). Madrid: Siglo XXI.

Juliá Díaz, S. (1995). Política en la Segunda República. Madrid: Marcial Pons.

Juliá DíAZ, S. (1986). "República, revolución y luchas internas". Anales de Historia de la Fundación Pablo Iglesias. vol 1. Madrid.

Juliá DíAZ, S. (1985). "Sindicatos, Partidos y Frente Popular”. Leviatán, segunda época. $\mathrm{n}^{\mathrm{o}} 22$.

JULIÁ DíAZ, S. (diciembre 1983). "Un líder político entre dirigentes sindicales". Revista del MOPU. $\mathrm{n}^{\circ} 305$.

LÓPEZ SANCHO, L. (28-XII- 1983). "Indalecio Prieto". A.B.C..

MACARRO VERA, J. M. (diciembre de 1982). "Causas de la radicalización socialista en la Segunda República". Revista de Historia Contemporánea. Sevilla. $n^{0} 1$.

MARÍN, M. A. (1-V-1983). "Una biografía incompleta" (Prieto). El País.

MARTÍNEZ REVERTE, J. (diciembre de 1983). "Un panfleto prietista". Revista del MOPU. $\mathrm{n}^{\mathrm{o}} 305$.

MASIP, A. (1981). Indalecio Prieto y Oviedo. Oviedo: Baraza Oviedo.

Miralles, R. (1988). "El particularismo político del socialismo vasco". Historia Contemporánea. UPV. $\mathrm{n}^{\mathrm{o}} 1$.

MirAlLes, R. (1988). El socialismo vasco durante la Segunda República. Bilbao: Universidad del País Vasco.

MORADIELlos, E. (1992). Las caras de Clio. Introducción a la Historia y a la Historiografia. Oviedo: Universidad de Oviedo.

MúgicA HerzoG, E. (mayo 1980). "Indalecio Prieto y el Partido Socialista". Sistema. $\mathrm{n}^{\mathrm{o}} 36$.

MúgiCA Herzog, E. (1-III-1984). "Prieto y el nacionalismo". El Correo españolEl Pueblo vasco.

OlABARRI GORTAZAR, I. (9- V- 1983). "Indalecio Prieto: el fracaso de un gran político". El Diario Vasco.

PASAMAR, G. (2000). La Historia contemporánea: Aspectos teóricos e historiográficos. Madrid: Editorial Síntesis. 
Perez Ledesma, M. (Domingo 6-VI-1982). "Por una nueva ruptura en la historia social". El País.

PRAT, J. (diciembre de 1983). "Acción y pasión en Indalecio Prieto". Revista del MOPU. $\mathrm{n}^{\mathrm{0}} 305$.

PRESTON, P. (diciembre de 1983). "Demócrata por encima de todo: Indalecio Prieto y la creación del Frente Popular". Revista del MOPU. $\mathrm{n}^{\mathrm{0}} 305$.

Preston, P. (1998). Las tres Españas del 36. Indalecio Prieto, una vida a la deriva. Barcelona: Plaza y Janés.

Preston, P. (2001). La Destrucción de la democracia en España. Barcelona: Grijalbo Mondadori.

REDERO SAN ROMÁN, M. (1983). "El movimiento socialista de la Segunda República en la historiografía de su cincuentenario". Studia Histórica, Historia Contemporánea. vol.1. $\mathrm{n}^{\mathrm{O}} 4$.

REDERO SAN ROMÁn, M. (1992). Estudios de Historia de la UGT. El movimiento socialista en la Segunda República (1931-1936). Salamanca: Ediciones Universidad de Salamanca.

SaIz Valdivielso, A. C. (1984). Indalecio Prieto: crónica de un corazón. Barcelona: Planeta.

Saiz Valdivielso, A. C. 1995). Los tres exilios de Indalecio Prieto. Madrid: Fundación españoles en el mundo.

TAIBÓ, F. I. "Prieto, contrabandista de armas". Historia 16. n 36.

TUÑón DE LARA, M. (1985). El movimiento obrero en la Historia de España. Madrid: Sarpe D.L..

TUÑ̃́N DE LARA, M. (1991). "El sistema de partidos en 1931-1933". Historia Contemporánea. UPV. $\mathrm{n}^{\circ} 6$.

TUÑón De LARA, M. (1976). La Segunda República. 2 vols. Madrid: Siglo XXI.

TUÑÓN DE LARA, M. (1990). "Transformaciones políticas e ideológicas de España durante el primer tercio del siglo XX". Historia Contemporánea. UPV. $\mathrm{n}^{\circ} 4$.

Velarde Fuentes, J. (1983). "Indalecio Prieto en Hacienda". Studia Histórica, Historia Contemporánea. vol. 1. $\mathrm{n}^{\mathrm{o}} 4$. 